\title{
Multiple roles of SOCS proteins: Differential expression of SOCS1 and SOCS3 in atherosclerosis
}

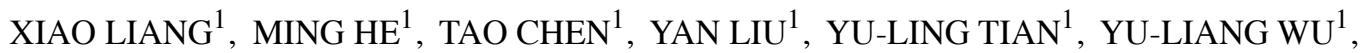 \\ YAN ZHAO $^{1}$, YAN SHEN ${ }^{1}$ and ZU-YI YUAN ${ }^{1-3}$ \\ ${ }^{1}$ Department of Cardiovascular Medicine, The First Affiliated Hospital of Medical School, Xi'an Jiaotong University; \\ ${ }^{2}$ Key Laboratory of Environment and Genes Related to Diseases, Xi'an Jiaotong University, Ministry of Education; \\ ${ }^{3}$ Key Laboratory of Molecular Cardiology, Xi'an Jiaotong University, Xi'an, Shaanxi 710061, P.R. China
}

Received November 29, 2012; Accepted February 1, 2013

DOI: $10.3892 /$ ijmm.2013.1323

\begin{abstract}
Pro-inflammatory cytokines play a key pathogenic role in atherosclerosis, which are induced by the Janus kinase/ signal transducer and activator of transduction (JAK/STAT) pathway. Furthermore, the JAK/STAT pathway is negatively regulated by the suppressor of cytokine signaling (SOCS) proteins. However, the change in SOCS expression levels and the correlation between SOCS expression and cholesterol levels in atherosclerosis is not yet well understood. To this end, a mouse model of atherosclerosis was established using apolipoprotein-deficient $\left(\mathrm{ApoE}^{-/-}\right)$mice. The mice were fed either a chow or high-fat diet. The mRNA and protein expression of SOCS1 and SOCS3 in plaque and vessels were determined at different time points. Furthermore, SOCS1 and SOCS3 mRNA expression was detected in the peripheral blood mononuclear cells (PBMCs) obtained from 18 male subjects with no coronary heart disease (non-CHD) population. The expression of SOCS1 in the $\mathrm{ApoE}^{-/-}$mice first increased and then decreased and the high-fat diet accelerated the appearance of the peak; the expression of SOCS3 increased with the increased feeding duration, and this trend was more pronounced in the mice fed the highfat diet. SOCS1/CD68 and SOCS3/CD68 showed opposite trends in expression with the increased duration of the highfat diet. Interleukin-6 (IL-6) expression in the main aorta of the $\mathrm{ApoE}^{-/-}$mice fed the high-fat diet also increased with the increased feeding duration. In the non-CHD population, the total serum cholesterol levels positively correlated with SOCS3 mRNA expression in the PBMCs $(r=0.433, \mathrm{P}=0.012)$.
\end{abstract}

Correspondence to: Professor $\mathrm{Zu}-\mathrm{Yi}$ Yuan, Department of Cardiovascular Medicine, The First Affiliated Hospital of Medical School, Xi'an Jiaotong University, 277 Yanta West Road, Xi'an, Shaanxi 710061, P.R. China

E-mail: zuyiyuan@mail.xjtu.edu.cn

Key words: atherosclerosis, inflammation, suppressors of cytokine signalling, macrophage, cholesterol
These results demonstrate the differential expression of SOCS1 and SOCS3 in atherosclerosis and suggest that SOCS3, together with IL-6 may promote the formation and development of atherosclerosis.

\section{Introduction}

Atherosclerosis is a chronic inflammatory disease that is associated with a variety of inflammatory cytokines and chemokines that control the balance of pro-inflammation and anti-inflammation $(1,2)$. This balance plays a critical role in the prognosis of atherosclerosis. Suppressor of cytokine signaling (SOCS) proteins are intracellular regulators of receptor signal transduction (3), which mainly regulate the Janus kinase/signal transducer and activator of transduction (JAK/STAT) pathway and play a regulatory role in the expression and activation of interleukin (IL)-6 (4), tumor necrosis factor- $\alpha$ (TNF- $\alpha$ ), as well as other inflammatory cytokines $(5,6)$.

SOCS protein expression closely correlates with the occurrence and development of inflammatory diseases through the regulation of gene expression and cellular activation, proliferation and differentiation (7). Previous studies have demonstrated that SOCS1 inhibits inflammation (8); SOCS1 reduces acute inflammation by regulating the activity of $\mathrm{T}$ cells (9), and $\mathrm{SOCS1}^{-/-}$mice suffer from fatal myocarditis (10). However, the role of SOCS3 remains controversial (11); intracellular protein therapy with SOCS3 has been shown to inhibit inflammation and apoptosis (12). SOCS3 transgenic mice have a Th2 response (13), while in mice with experimental autoimmune encephalomyelitis, inflammation was attenuated upon the intravenous injection of SOCS3 $3^{-/}$dentritic cells (14). In addition, bone marrow-derived macrophages differentiate into the $\mathrm{M} 2$ phenotype in $\mathrm{SOCS}^{-/-}$rats, even under the conditions of classic activation (15). Macrophages express different SOCS proteins under various stimulatory conditions. Interferon- $\gamma$ mainly induces SOCS1 expression (16) and interleukins, such as IL-6 and IL-10, induce SOCS3 expression (17). In the SOCS protein family, different subtypes play different roles in inflammatory diseases $(18,19)$.

Hyperlipidemia, particularly hypercholesterolemia, is the most recognized risk factor for atherosclerosis (20). Circulating 
cholesterol may cause endothelial cell dysfunction on its own or by its chemical modifications, thus affecting the chemotaxis and adhesion of leukocytes and the release of several inflammation-activating cytokines (21-23), which further promotes the deposition of cholesterol under the arterial intima. The interaction between cholesterol and inflammation occurs throughout the onset and development of atherosclerosis (24). However, the mechanisms by which hypercholesterolemia influences SOCS expression and the ensuing effects (i.e., atherosclerosis) remain unclear. Through observations and quantitative analysis of the expression of SOCS1 and SOCS3 in atherosclerotic plaque in apolipoprotein-deficient $\left(\mathrm{ApoE}^{-/}\right)$mice at different intervention time points, this study aimed to clarify the trends and possible mechanisms of action of SOCS1 and SOCS3 proteins in the formation of atherosclerotic plaque following exposure to high cholesterol levels, and to investigate the correlation between blood cholesterol levels and the expression of SOCS1 and SOCS3. The objective of this study was to further clarify the role of SOCS1 and SOCS3 in the occurrence and development of atherosclerosis.

\section{Materials and methods}

Animals. The animal experiments were carried out in accordance with the Guide for the Care and Use of Laboratory Animals published by the US National Institutes of Health (NIH Publication no. 85-23, revised 1996). The ApoE ${ }^{-/}$mice were kept under constant temperature conditions $\left(18^{\circ} \mathrm{C}\right)$ with a 12-h light-dark cycle (light from 8:00 a.m. to 8:00 p.m.) and allowed free access to food and water; these mice were a gift from Dr Edward M. Rubin at the University of California, Berkeley (Berkeley, CA, USA). C57BL/6j mice were purchased from the Fourth Military Medical University, Xi'an, China. $\mathrm{C} 57 \mathrm{BL} / 6 \mathrm{j}$ and $\mathrm{ApoE}^{-/}$mice were fed a chow diet (4\% fat and $0 \%$ cholesterol) after being weaned at 3 weeks of age, then half of the $\mathrm{ApoE}^{-/-}$mice were switched to a high-fat diet containing $21 \%$ fat and $0.15 \%$ cholesterol from 6 weeks of age. The C57BL/6j mice were sacrificed at 12, 20 and 28 weeks, while the $\mathrm{ApoE}^{-/}$ mice were sacrificed at 12, 16, 20, 24 and 28 weeks (Fig. 1). The protocols for the experiments (sacrifice, blood and main aorta harvest) were approved by the Institutional Ethics Committee for Animal Experiments of Xi'an Jiaotong University, Xi'an, China and the mice were sacrificed after anesthesia, as previously described (25).

Clinical trials. Subjects included 18 male patients who were 35-45 years of age and recruited from the First Affiliated Hospital of the Medical College of Xi'an Jiaotong University. Coronary heart disease (CHD) was diagnosed by the American Heart Association (AHA) 2007 criteria (26). Subjects who were suffering from $\mathrm{CHD}$, hypertension, diabetes, acute or chronic infection, fever, cancer, autoimmune disease, severe liver, kidney or other major organ diseases, had surgery within the last 2 weeks, or were taking anti-inflammatory drugs and/or immune inhibitors during the month preceding the study, were excluded. Informed consent was obtained from all subjects. The study was carried out according to the principles outlined in the Declaration of Helsinki and the study protocol was approved by the Xi'an Jiaotong University Ethics Committee. Peripheral blood mononuclear cells (PBMCs) were separated by density gradient centrifugation with lymphocyte isolation solution (Shanghai Huajing Biotech Co., Shanghai, China) and stored at $-80^{\circ} \mathrm{C}$.

Detection of lipid and glucose in serum. Serum was separated by the centrifugation of venous blood from mice and patients at 3,000 rpm for $15 \mathrm{~min}$, and total cholesterol (TC), triglyceride (TG), high-density lipoprotein cholesterol (HDL-C) and lowdensity lipoprotein cholesterol (LDL-C) levels were measured using assay kits (Dongou Bioengineering, Beijing, China) while glucose levels were measured using an Accu-Chek1 Advantage Glucometer (Roche Diagnostics, Inc., Indianapolis, IN, USA), according to the manufacturer's instructions. Each sample was examined 3 times.

Chemical and immunohistochemical staining. The root of the aorta was dissected under a microscope and frozen in optimal cutting temperature embedding medium for serial cryosectioning at $6 \mathrm{~mm}$, covering $500 \mathrm{~mm}$ of the root. The first section was harvested when the 3 aortic valve cusps became visible in the lumen of the aorta, and every 5 th section was harvested on 1 slide (8 sections/slide). Oil Red O staining was used to detect lipids in the plaque and the sections were analyzed using polarization microscopy. Immunohistochemistry was performed with antibodies against SOCS1 (1:100; Abcam, Cambridge, MA, USA) and SOCS3 (1:50; Santa Cruz Biotechnology, Inc., Santa Cruz, CA, USA). Negative controls in the absence of primary antibodies were also used. Section images were captured digitally using an Olympus BX51 imaging system and were quantified with Image-Pro Plus 6.0 software. The crosssectional surface area of the lesion and total cross-sectional vessel area were also quantified, as previously described (27).

RNA extraction and quantitative PCR. SOCS1 and SOCS3 mRNA expression in the main aorta of the mice and in the PBMCs of patients were determined using quantitative PCR. Total RNA was isolated from the main aorta of the mice and PBMCs of patients with TRIzol reagent (Invitrogen, Carlsbad, CA, USA); the NanoDrop 1000 spectrophotometer (Thermo Scientific) was used to quantify the total RNA. The resulting RNA was reverse transcribed and analyzed by quantitative PCR using the SYBR PrimeScript ${ }^{\mathrm{TM}}$ RT-PCR Kit II (Takara Bio, Inc.). All real-time reactions were performed on the iQ5 $^{\text {TM }}$ Multicolor Real-time PCR detection system (Bio-Rad, Hercules, CA, USA). A three-step PCR procedure of $5 \mathrm{sec}$ at $95^{\circ} \mathrm{C}, 20 \mathrm{sec}$ at $63.5^{\circ} \mathrm{C}$ and $10 \mathrm{sec}$ at $72^{\circ} \mathrm{C}$ was applied for 45 cycles. GAPDH was used as a housekeeping gene. The primer sequences are shown in Table I. The data were analyzed using the $2^{-\Delta \Delta C T}$ method, as previously described (28). Each sample was examined 3 times.

Western blot analysis. Proteins from the main aorta were extracted by RIPA buffer (Cybrdi, Inc.) according to the manufacturer's instructions, and a protease inhibitor cocktail (Roche Diagnostics, Inc.) was added to all samples. The BCA protein assay reagent kit (Pierce) was used to quantify the total amount of protein. Equal amounts of protein extracts were separated by $10 \%$ SDS-PAGE gel and then transferred onto nitrocellulose membranes using a Bio-Rad transfer blotting system (Bio-Rad). Skim milk of 5\% was used for blocking non-specific binding for $1 \mathrm{~h}$ at room temperature with slow 
Table I. Primers used for real-time PCR.

\begin{tabular}{llll}
\hline Gene name & \multicolumn{1}{c}{ Forward (5'-3') } & \multicolumn{1}{c}{ Reverse (5'-3') } & NM code \\
\hline Mouse & & & \\
GAPDH & TCAACGGCACAGTCAAGG & ACTCCACGACATACTCAGC & NM_008084.2 \\
SOCS1 & TCCGATTACCGGCGCATCACG & CTCCAGCAGCTCGAAAAGGCA & NM_009896.2 \\
SOCS3 & CACAGCAAGTTTCCCGCCGCC & GTGCACCAGCTTGAGTACACA & NM_007707.2 \\
IL-6 & AGCCAGAGTCCTTCAGAGAGATAC & GCTAAGGACCAAGACCATCCAATT & NM_031168.1 \\
TNF- $\alpha$ & GCTCTTCTGTCTACTGAACTTCGG & CCAGACCCTCACACTCAGATCAT & NM_013693.2 \\
TNF- $\beta$ & GCAACATGTGGAACTCTACCAGAA & GACGTCAAAAGACAGCCACTCA & NM_011577.1 \\
Human & & & \\
GAPDH & AACATCATCCCTGCCTCTACTG & CTCCGACGCCTGCTTCACC & NM_002046.3 \\
SOCS1 & GCAGCCGACAATGCAGTCT & CGAACGGAATGTGCGGAAGTG & NM_003745.1 \\
SOCS3 & GCCACCTACTGAACCCTCCTC & TCCGACAGAGATGCTGAAGAGTG & NM_003955.3 \\
\hline
\end{tabular}

SOCS, suppressor of cytokine signaling; IL-6, interleukin-6; TNF- $\alpha$, tumor necrosis factor- $\alpha$.

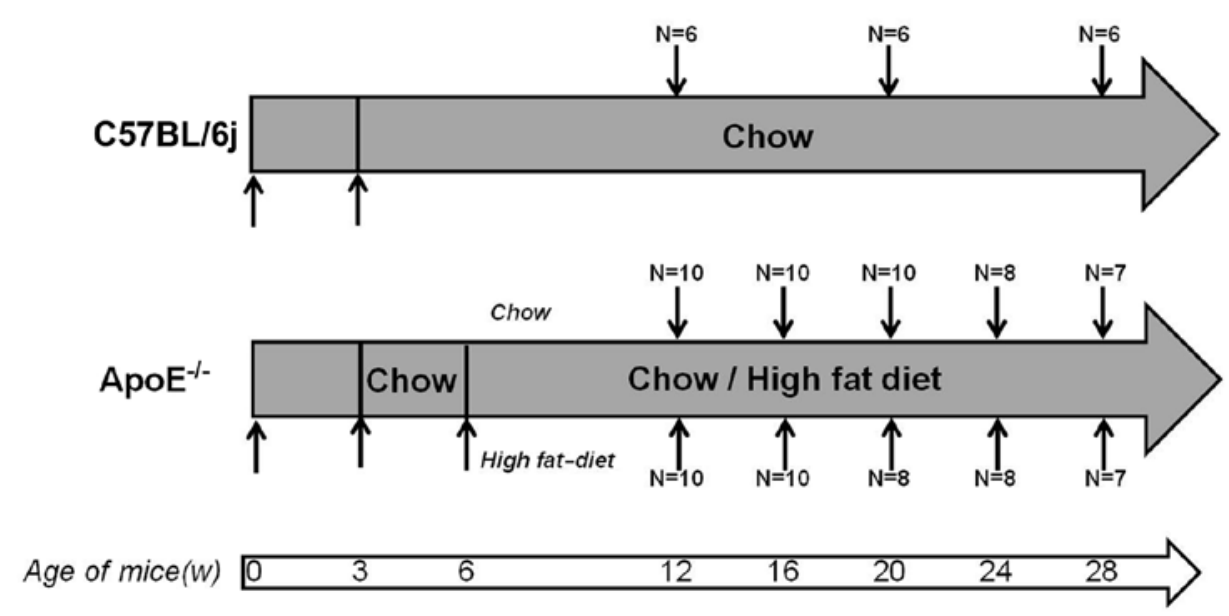

Figure 1. Time and feeding schedule of the animal model. w, weeks; N, number of mice.

shaking. The blots were incubated overnight at $4^{\circ} \mathrm{C}$ with anti-SOCS3 (1:500; Santa Cruz Biotechnology, Inc.), antiSOCS1 (1:500; Abcam) or anti-GAPDH (1:1,000; Santa Cruz Biotechnology, Inc.). A horseradish peroxidase-conjugated anti-goat (1:10,000; Abcam) or anti-rabbit secondary antibody (1:5,000; Abcam) and enhanced chemiluminescent substrate (Pierce) were used for detection, as previously described (29). Each sample was examined 3 times.

Statistics analysis. Data were expressed as the means \pm SD. The Student's t-test or one-way ANOVA were used to analyze the differences among groups. Post hoc comparisons between groups were performed using the Newman-Keuls multiple comparison test. A value of $\mathrm{P}<0.05$ was considered to indicate a statistically significant difference.

\section{Results}

General condition of the experimental animals. After being fed with different diets for 6 weeks, the total serum cholesterol levels of the $\mathrm{ApoE}^{-/-}$mice fed a high-fat diet were significantly higher than those of the mice fed the chow diet (2.52-fold) and the $\mathrm{C} 57 \mathrm{Bl} / 6 \mathrm{j}$ mice (31.05-fold) $(\mathrm{P}<0.01)$; however, the total serum cholesterol levels did not change significantly with the prolonged feeding time in each group. The TG, HDL-C and LDL-C levels in the serum of the ApoE $\mathrm{E}^{-/-}$mice were higher than those in the serum of the $\mathrm{C} 57 \mathrm{BL} / 6 \mathrm{j}$ mice $(\mathrm{P}<0.01)$; however, the $\mathrm{ApoE}^{-/-}$mice with different dietary interventions showed no significant differences in the aforementioned indexes. Body weight increased significantly in each group with the increased feeding duration. No significant difference in serum glucose levels was observed among the groups (Table II).

Positive Oil Red O staining showed that the atherosclerotic plaque size at the aortic root and lipid deposition in the plaque of the $\mathrm{ApoE}^{-/-}$mice were consistently growing as the feeding duration increased. At 28 weeks, the Oil Red O positive area was 4.33-fold larger than that at 12 weeks in the group fed the chow diet $(\mathrm{P}<0.05)$ and 3.62-fold larger in the group fed the high-fat diet $(\mathrm{P}<0.01)$. The area of lipid deposit in the atherosclerotic plaque in the group fed the high-fat diet was larger than that in the group fed the chow diet in $\mathrm{ApoE}^{-/-}$mice of the same age (Fig. 2). 


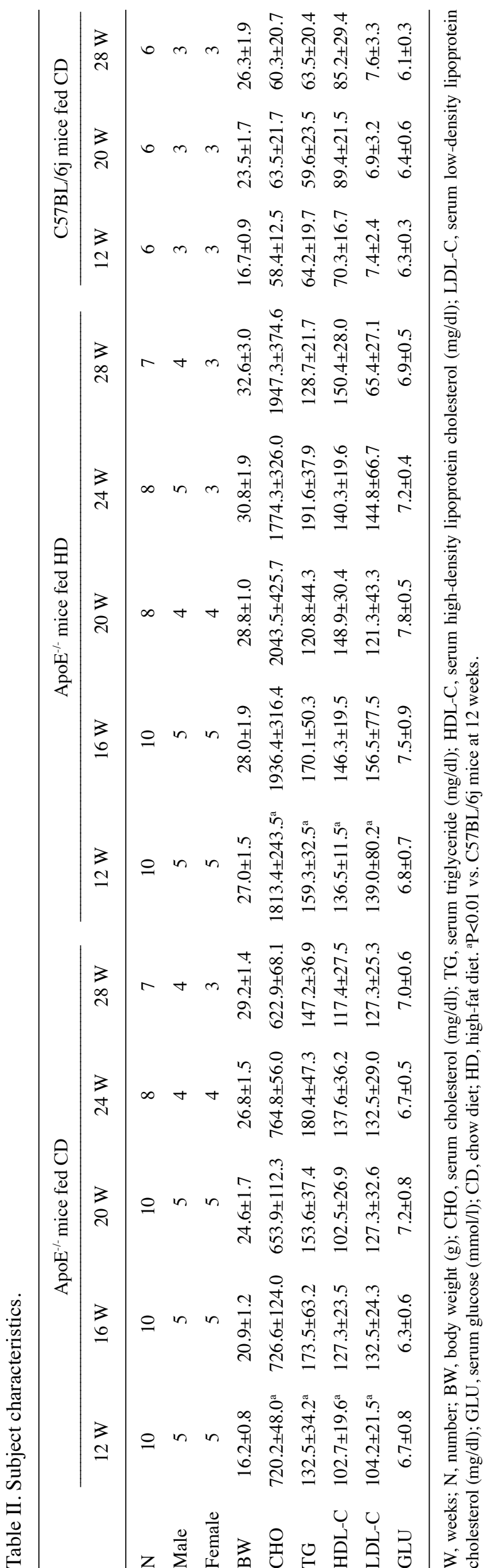

Trend in SOCS1 expression in the ApoE ${ }^{-/-}$mice. The results of immunohistochemical staining revealed that in the group fed the chow diet, SOCS1 expression in the plaque at the aortic root of the ApoE ${ }^{-/}$mice showed an increasing trend first (eventually peaking at 24 weeks) and then decreased as the feeding duration increased $(\mathrm{P}<0.05)$. The same single peak trend appeared earlier in the group fed the high-fat diet at 20 weeks $(\mathrm{P}<0.05)$ (Fig. 3A, B and E). Surprisingly, the results of the real-time PCR and western blot analyses after the extraction of RNA and protein levels showed a similar trend in the expression of SOCS1 in the animals fed the different diets. In the group fed the chow diet, the SOCS1 protein expression level was 11.85-fold higher and the mRNA expression level was 3.68 -fold higher at 24 weeks than at 12 weeks $(\mathrm{P}<0.01)$ (Fig. 4A and B). In the group fed the high-fat diet, the SOCS1 protein expression level was 4.32-fold higher and the mRNA expression level was 2.02-fold higher at 20 weeks than at 12 weeks $(\mathrm{P}<0.01)$ (Fig. 4C).

Upregulation of SOCS3 in the ApoE ${ }^{-/}$mice. SOCS1 and SOCS3 belong to the SOCS family of proteins. To confirm whether they have the same expression tendency in $\mathrm{ApoE}^{-/-}$ mice, we also detected the SOCS3 mRNA and protein level. Immunohistochemical staining showed that in the groups fed the chow and high-fat diet, the SOCS3-positive areas within the plaque of the $\mathrm{ApoE}^{-/}$mice were enlarged with the increased feeding duration. The results of the real-time PCR and western blot analyses were consistent with the immunohistochemical results. The expression of SOCS3 mRNA and protein in the aorta of the $\mathrm{ApoE}^{-/}$mice increased significantly after 20 weeks, regardless of the type of diet, and at 28 weeks, the expression of SOCS3 mRNA had significantly increased $(\mathrm{P}<0.01)$ (Figs. 3C, D and F and Fig. 4). At the same time point, the SOCS3 expression level was higher in the group fed the high-fat diet than in the group fed the chow diet. The differential expression of SOCS1 and SOCS3 suggests that they play different roles in atherosclerosis.

Expression levels of SOCS1 and SOCS3 in the aorta of $\mathrm{C} 57 \mathrm{Bl} / 6 \mathrm{j}$ mice. ApoE ${ }^{-/}$mice are used in classic animal models of atherosclerosis, due to hyperlipidemia. We also used a wild-type control to identify the correlation between blood lipids and inflammatory cytokines. No formation of plaque was found in the aortic root in the $\mathrm{C} 57 \mathrm{Bl} / 6 \mathrm{j}$ mice of different ages in the group fed the chow diet (12, 20 and 28 weeks), and Oil Red $\mathrm{O}$ staining displayed no lipid deposition (data not shown). Immunohistochemical staining showed that the expression of SOCS1 and SOCS3 at the aortic root of C57Bl/6j mice of different ages was very limited (Fig. 5A). Real-time PCR conducted with RNA extracted from the aorta of C57Bl/6j mice revealed that the SOCS1 and SOCS3 expression level was $22 \%$ and $9 \%$ in the $\mathrm{ApoE}^{-/-}$mice in the group fed the chow diet at 12 weeks, respectively $(\mathrm{P}<0.01)$ (Fig. 5B).

SOCS1/CD68 and SOCS3/CD68 show opposite trends in expression with the increasing feeding duration of the high-fat diet. As SOCS proteins are expressed in several cell types but mainly in macrophages, which induce the majority of inflammatory cytokines in disease, we detected the ratio of SOCS1/3 and macrophages (CD68) to clarify the contribution of macrophages 


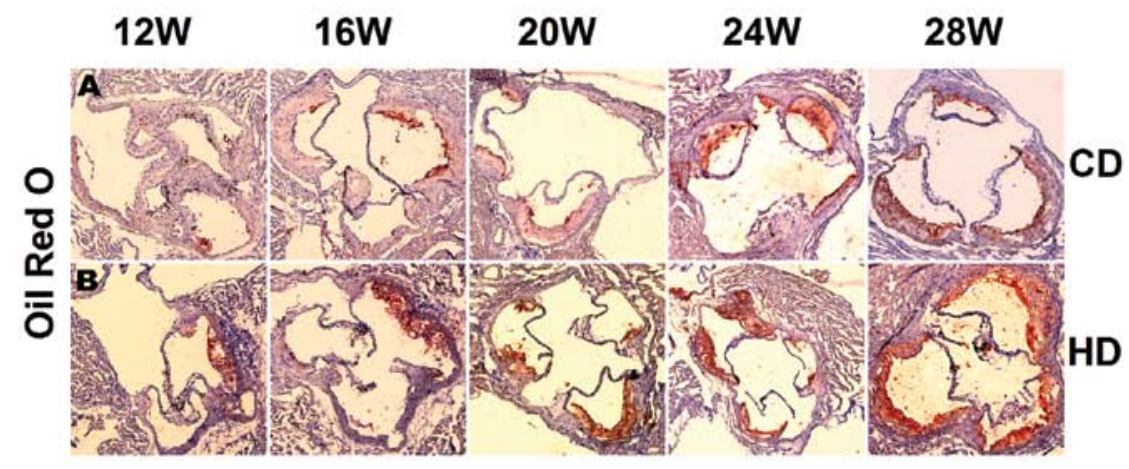

C ORO Staining in ApoE-1- Mouse Plaque

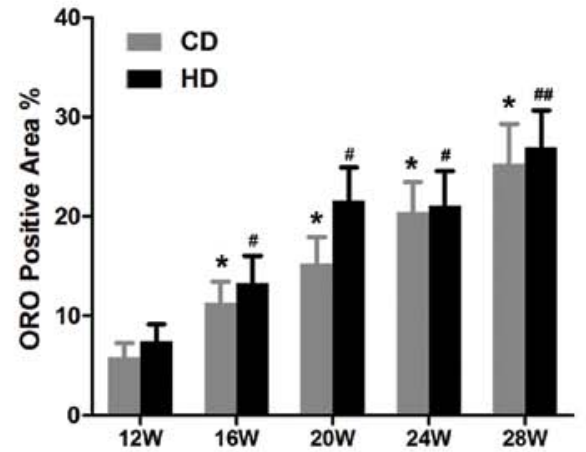

Figure 2. Oil Red $\mathrm{O}(\mathrm{ORO})$ staining to detected lipid deposit in atherosclerotic plaque in $\mathrm{ApoE}^{-/}$mice at different time points. (A and $\left.\mathrm{B}\right) \mathrm{ORO}$ staining in plaque of $\mathrm{ApoE}^{-/}$mice fed the chow and high-fat diet; magnification, $\mathrm{x} 100$; bar, $200 \mu \mathrm{m}$. (C) ORO staining positive rate in plaque of ApoE $\mathrm{E}^{-/} \mathrm{mice}$ fed the chow and high-fat diet. The bars represent the means $\pm \mathrm{SD}$. W, weeks; $\mathrm{CD}$, chow diet, $\mathrm{HD}$, high-fat diet. ${ }^{*} \mathrm{P}<0.05$ vs. mice fed the chow diet for 12 weeks; ${ }^{\#} \mathrm{P}<0.05$ vs. mice fed the high-fat diet for 12 weeks; ${ }^{\# /} \mathrm{P}<0.01$ vs. mice fed the high-fat diet for 12 weeks.

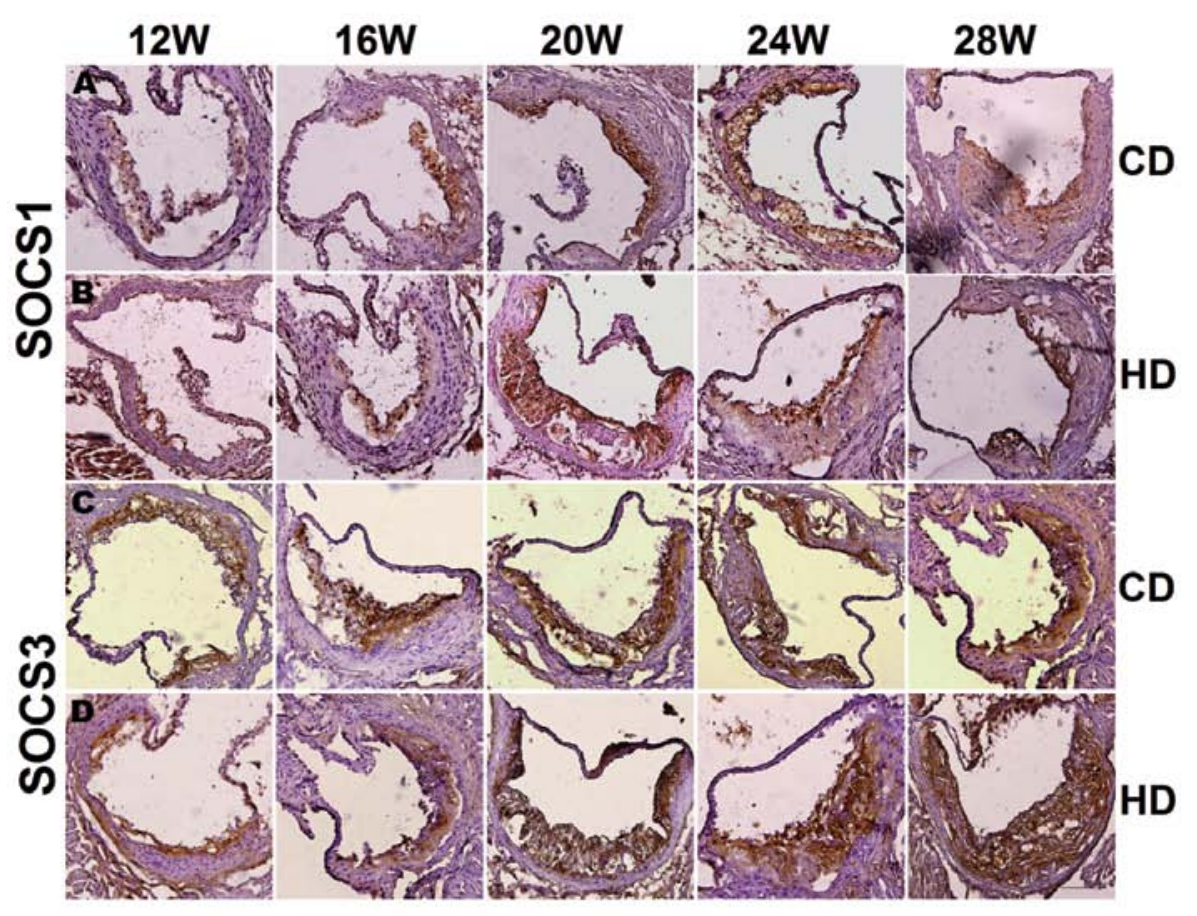

E Socs1 Expression in ApoE'-Plaque

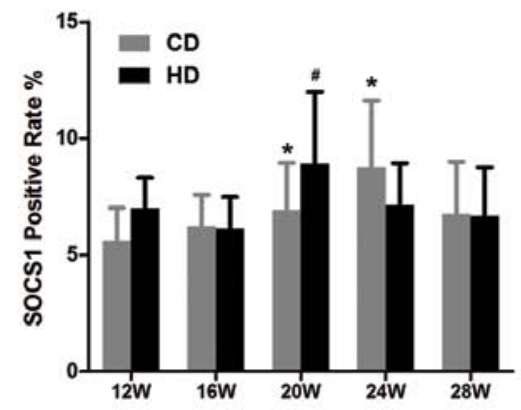

F socs3 Expression in ApoE*-Plaque

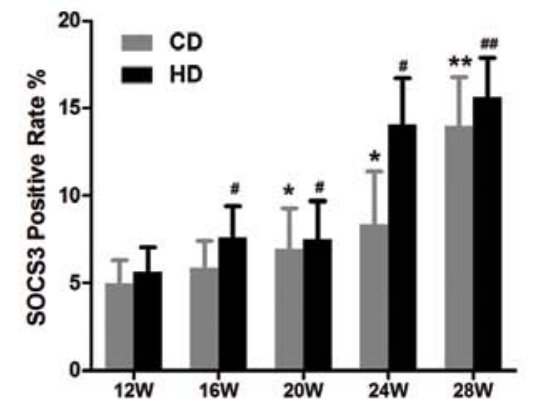

Figure 3. Immunohistochemistry for the detection of the expression of SOCS1 and SOCS3 in plaque of AopE ${ }^{-/}$mice at different time points. (A-D) Expression of SOCS1 and SOCS3 in plaque of ApoE ${ }^{-/}$mice fed the chow and high-fat diet; magnification, $\mathrm{x} 200$; bar, $100 \mu \mathrm{m}$. (E and F) SOCS1 and SOCS3 positive rate in plaque of $\mathrm{ApoE}^{-/}$mice fed the chow and high-fat diet. The bars represent the means $\pm \mathrm{SD}$. W, weeks; $\mathrm{CD}$, chow diet, $\mathrm{HD}$, high-fat diet. "P<0.05 vs. mice fed the chow diet for 12 weeks; ${ }^{* *} \mathrm{P}<0.01$ vs. mice fed the chow diet for 12 weeks; ${ }^{\prime} \mathrm{P}<0.05$ vs. mice fed the high-fat diet for 12 weeks; ${ }^{\# / t} \mathrm{P}<0.01$ vs. mice fed the high-fat diet for 12 weeks.

in disease progression. According to the immunohistochemical staining results of the $\mathrm{ApoE}^{-/-}$mice fed the high-fat diet, the CD68-positive area in the aortic root plaque increased in size as the feeding period increased, and the ratio of the SOCS1/CD68 stained areas decreased gradually over time (from $0.68 \pm 0.32$ at 12 weeks to $0.39 \pm 0.21$ at 28 weeks, $\mathrm{P}<0.05$ ). The ratio of the stained area of SOCS3/CD68 showed an inverse trend as compared to SOCS1 $(0.56 \pm 0.32$ at 12 weeks to $0.73 \pm 0.42$ at 28 weeks, $\mathrm{P}<0.05$ ) (Fig. 5C). These results suggest that SOCS1 and SOCS3 play different roles in disease progression.
Expression of inflammatory cytokines in vascular tissue of ApoE ${ }^{-/-}$mice. It is well known that SOCS protein expression correlates with the expression of inflammatory cytokines. To confirm whether a correlation exists between SOCS3 and IL-6 expression in atherosclerosis, as shown in other diseases, we detected the expression levels of a number of inflammatory cytokines. As the high-fat diet feeding duration increased, the expression levels of IL-6 mRNA in the aorta of the ApoE ${ }^{-1-}$ mice increased continuously (the expression levels at 28 weeks were 40.64 -fold higher than those at 12 weeks), whereas no 
A
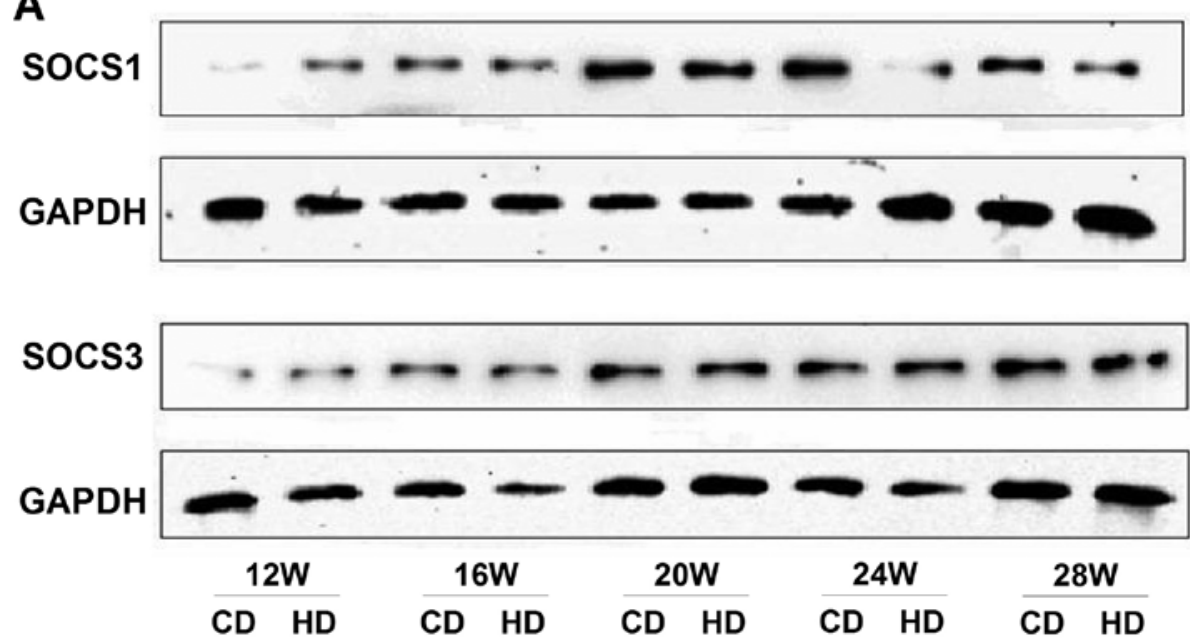

B

SOCS1 Expression in ApoE*- Vessel

SOCS3 Expression in ApoE ${ }^{+-}$Vessel
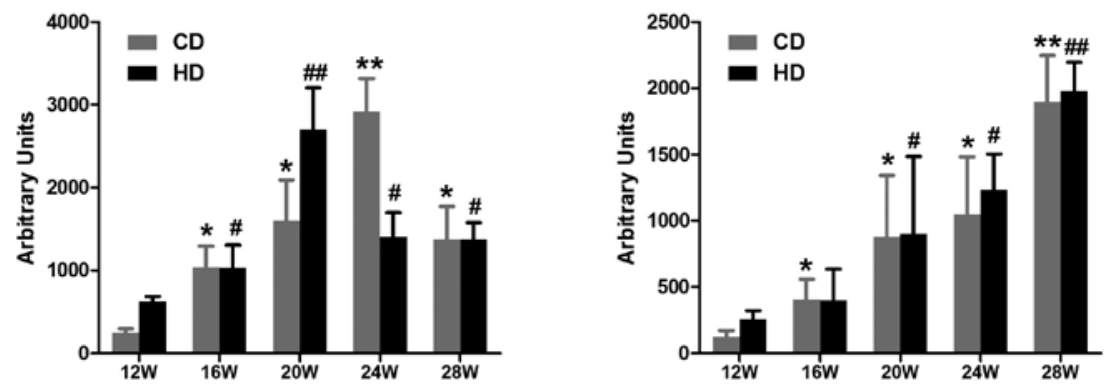

C SOCS1 mRNA Expression in ApoE*- Vessel

SOCS3 mRNA Expression in ApoE ${ }^{-1}$ Vessel
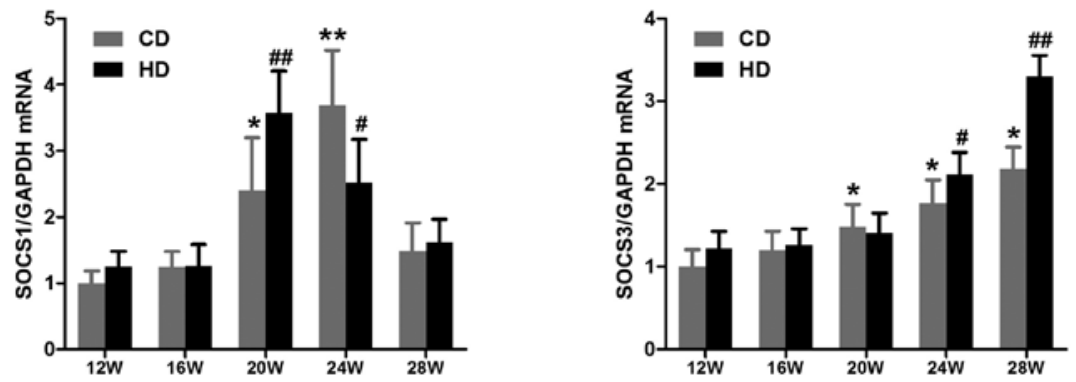

Figure 4. Protein and mRNA expression of SOCS1 and SOCS3 in main aorta of $\mathrm{ApoE}^{-/}$mice fed the chow and high-fat diet. (A and B) Protein expression of SOCS1 and SOCS3 in the main aorta of ApoE ${ }^{-/}$mice fed the chow and high-fat diet. (C) mRNA expression of SOCS1 and SOCS3 in the main aorta of ApoE ${ }^{-/}$mice $^{-1}$ fed the chow and high-fat diet. The bars represent the means \pm SD. W, weeks; CD, chow diet, HD, high-fat diet. GAPDH was detected as an internal reference. ${ }^{\text {"P}} \mathrm{P}<0.05$ vs. mice fed the chow diet for 12 weeks; ${ }^{* *} \mathrm{P}<0.01$ vs. mice fed the chow diet for 12 weeks; ${ }^{*} \mathrm{P}<0.05$ vs. mice fed the high-fat diet for 12 weeks; ${ }^{\# \#} \mathrm{P}<0.01$ vs. mice fed the high-fat diet for 12 weeks.

change in the mRNA expression of TNF- $\alpha$ was observed (Fig. 5D and E).

Positive correlation between total serum cholesterol levels and SOCS3 mRNA expression in PBMCs of the non-CHD population. To confirm our above results, we also investigated the correlation between SOCS expression and cholesterol levels in humans. In the PBMCs of the non-CHD population $(n=18$, males, 35-45 years of age), a positive correlation was observed between the total serum cholesterol levels and SOCS3 mRNA expression ( $\mathrm{r}=0.433, \mathrm{P}=0.012$ ) (Fig. 6). However, no correlation was observed between the total serum cholesterol levels and the expression of SOCS1 mRNA in the PBMCs $(\mathrm{r}=0.061, \mathrm{P}=0.45)$. Further analysis revealed no correlation between the expression of SOCS1 and SOCS3 mRNA, age, body mass index (BMI) and other factors $(\mathrm{P}>0.05)$ (data not shown).

\section{Discussion}

In the present study, we investigated SOCS1 and SOCS3 expression in $\mathrm{ApoE}^{-/}$mice exposed to high levels of cholesterol for increasing periods of time. SOCS1 expression showed a single peak change, whereas SOCS3 expression showed a continuous increase. In the animals exposed to different levels of cholesterol, high cholesterol levels increased SOCS1 and SOCS3 expression in $\mathrm{ApoE}^{-/}$mice of the same age but had no effect on the respective change oinf SOCS1 and SOCS3 expression with the increased feeding duration. IL-6 expression 


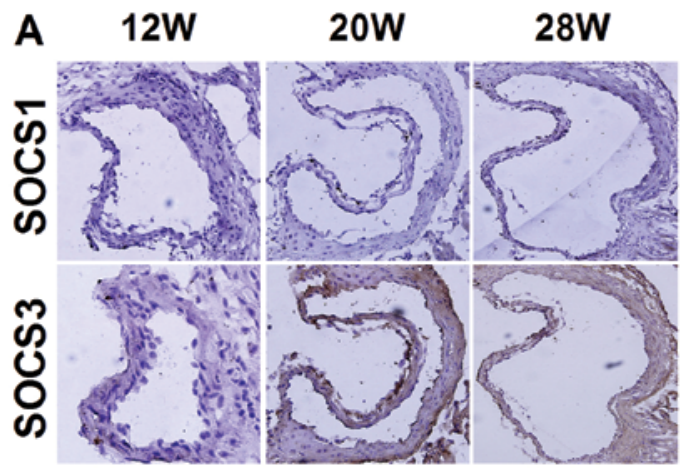

B SOCS mRNA Expression in Vessel
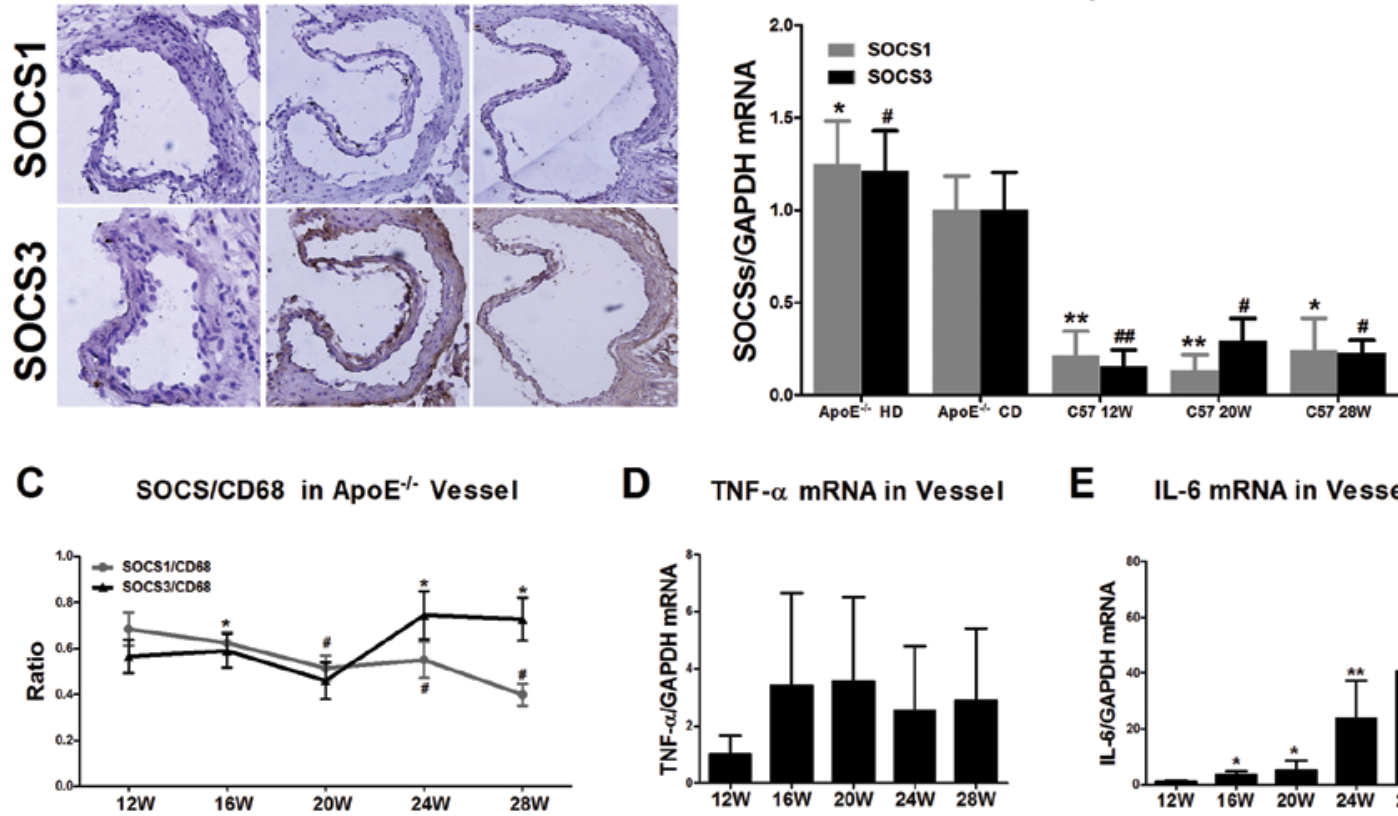

D TNF- $\alpha$ mRNA in Vessel

E IL-6 mRNA in Vessel
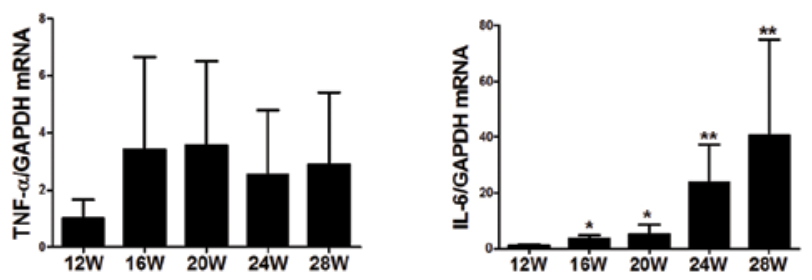

Figure 5. Other information about SOCS expression in mice. (A) Immunol histochemistry was used to detect the positive rate of SOCS1 and SOCS3 in plaque of C57BL/6j mice fed the chow diet; magnification, x200; bar, $100 \mu \mathrm{m}$. (B) mRNA expression of SOCS1 and SOCS3 in the main aorta in 12 week-old-ApoE ${ }^{-1}$ mice fed the high-fat diet and in C57BL/6j mice. CD, chow diet; HD, high-fat diet. (C) The positive rate of SOCS/CD68 in plaque of ApoE ${ }^{-1-}$ mice fed the high-fat diet. " $\mathrm{P}<0.05$ vs. mice fed the chow diet for 12 weeks; ${ }^{* *} \mathrm{P}<0.01$ vs. mice fed the chow diet for 12 weeks (SOCS1); ${ }^{*} \mathrm{P}<0.05$ vs. mice fed the chow diet for 12 weeks; ${ }^{\# \#} \mathrm{P}<0.01$ vs. mice fed the chow diet for 12 weeks (SOCS3). (D and E) TNF- $\alpha$ and IL-6 mRNA expression in main aorta of ApoE ${ }^{-/}$mice the high-fat diet. ${ }^{*} \mathrm{P}<0.05$ vs. mice fed the high-fat duet for 12 weeks; ${ }^{* *} \mathrm{P}<0.01$ vs. mice fed the high-fat duet for 12 weeks. The bars represent the means $\pm \mathrm{SD}$. W, weeks.

The Correlation Between CHO and SOCS3 mRNA in Non-CHD Population PBMC

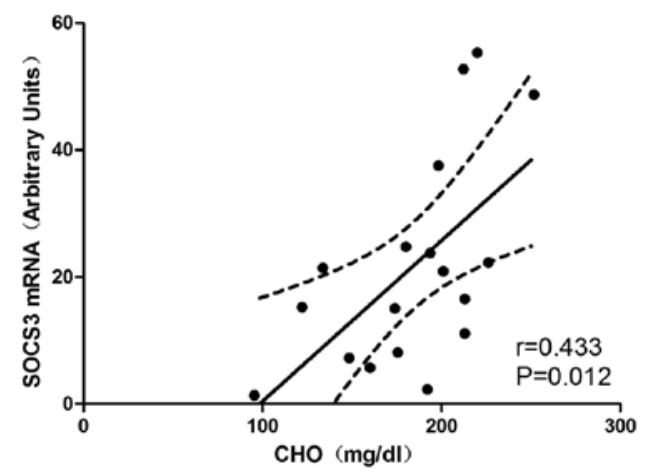

Figure 6. The correlation between total serum cholesterol (CHO) levels and SOCS3 mRNA in peripheral blood mononuclear cells (PBMCs) from the noncoronary heart disease (CHD) population.

in the aortas of the $\mathrm{ApoE}^{-/}$mice increased with the increase in SOCS3 expression. In addition, SOCS3 mRNA expression positively correlated with cholesterol levels in the non-CHD population. In conclusion, SOCS1 and SOCS3 expression differ in $\mathrm{ApoE}^{-/-}$mice with the progression of the disease and SOCS3 may play a pro-atherosclerotic role.

In this study, the trend in SOCS1 and SOCS3 expression, which was similar to that shown in the study by Tang et al (30), demonstrated that although these 2 proteins belong to the SOCS family, they play opposing roles in the initiation and development of atherosclerosis. According to previous studies, the existence of the $\mathrm{SH} 2$ domain in the SOCS protein family may compete with JAK to bind STAT, thus inhibiting the phosphorylation of STAT and subsequent inflammatory response (31). As a result, studies on the SOCS protein family have focused on inflammatory disease or a variety of inflammatory pathways (32). Previous studies have demonstrated a consistent view on the role of SOCS1 (33), which has been shown to inhibit the progression of inflammatory disease. However, the role of SOCS3 remains controversial, as described in the Introduction. The trend in the expression of SOCS1 which exerts an anti-inflammation effect was consistent with that of pathophysiological processes. Although the role of SOCS3 in atherosclerosis remains controversial, the results of this study revealed that SOCS3 may play the following roles in atherosclerosis: i) SOCS3 may exert a pro-inflammatory effect, which is consistent with the progression of the disease; its expression increased with increasing cholesterol levels and the prolonged feeding duration; ii) SOCS3 may play multiple roles in atherosclerosis and other inflammatory diseases; in various types of inflammatory diseases, the reflected net effect may differ or involve a more complex mechanism of immune regulation; thus, its role cannot simply be explained as a balance of anti-inflammation and pro-inflammation; iii) SOCS3 is expressed during the late stages of atherosclerotic lesions, indicating that it plays an important role in the late stages of the disease and may play a crucial role in maintaining plaque stability, which requires further validation studies; and iv) SOCS3 expression may be subjected to the regulation of other inflammatory factors during the progression of atherosclerosis. When the pro-inflammatory/ anti-inflammatory balance tilted in favor of pro-inflammation, the expression of SOCS3 also increased. In the long process of the onset and development of human atherosclerosis, immune- 
inflammatory mechanisms play an important role, and both pro-inflammatory and anti-inflammatory factors are present in this complex network (34). Overall, SOCS proteins ultimately inhibit the progression of inflammation by inhibiting the phosphorylation of STAT, and the JAK/STAT pathway influences the progression of atherosclerosis. Based on the results obtained in this study, we hypothesized that SOCS1 and SOCS3, through changes in their expression, affect the downstream signaling pathway, thus influencing the development of atherosclerosis. Upon exposure to high cholesterol levels, these 2 factors show differential expression patterns, suggesting that during the development of atherosclerosis, SOCS1 and SOCS3 may play different roles in addition to the common mechanisms of SOCS proteins (i.e., SOCS1 has a clear anti-inflammatory effect while SOCS3 may play a pro-inflammatory role).

The SOCS protein family is mainly expressed in activated macrophages (3), and under the induction of high cholesterol, the number of macrophages in the atherosclerotic plaque of $\mathrm{ApoE}^{-/-}$mice continuously increased. Descriptive studies on cholesterol and SOCS expression in disease are limited. During the prolonged high cholesterol exposure in our study, the differential expression of SOCS1 and SOCS3 was independent of the changes in the number of macrophages. The expression of anti-inflammatory SOCS1 per macrophage decreased with the increase in the exposure time to high cholesterol levels, while an opposite trend was observed with SOCS3. The difference in the expression trends of these 2 proteins further suggests that the 2 proteins play different roles in the occurrence and development of atherosclerosis. If these 2 factors antagonize each other, then the expression of SOCS1, with anti-inflammatory effects, would gradually decrease, while that of SOCS3, which may have pro-inflammatory effects, would steadily increase in line with the accelerated disease process. These different trends were mainly observed in the differential expression patterns of these 2 proteins in plaque.

In the $\mathrm{C} 57 \mathrm{Bl} / 6 \mathrm{j}$ mice of the control group, the expression of SOCS1 and SOCS3 in the aorta remained low, and no plaque was generated, regardless of the age of the mice. In the $\mathrm{ApoE}^{-/}$ mice, even when those fed a normal diet, the total serum cholesterol level was 10 -fold higher than that in the $\mathrm{C} 57 \mathrm{Bl} / 6 \mathrm{j}$ mice; when the ApoE ${ }^{-/}$mice were fed the high-fat diet, their plasma cholesterol level increased by $>29$-fold compared to the $\mathrm{C} 57 \mathrm{Bl} / 6 \mathrm{j}$ mice. This indicates that high cholesterol is not only an important factor leading to the generation of atherosclerotic plaque, but also an important condition that induces the expression of SOCS1 and SOCS3 proteins. This also indicates that the changes in SOCS1 and SOCS3 expression over time are related to the exposure to a high-fat diet as opposed to age. Hypercholesterolemia plays a crucial role in the occurrence and development of atherosclerosis, promotes subintimal lipid deposition, and this metabolic disorder also contributes to immune-inflammatory response changes in the body. The change in the metabolic level, represented by high levels of cholesterol, affects a variety of cytokines and chemotactic proteins that are involved in the inflammatory response and alters the inflammatory network equilibrium, which affects the speed of subendocardial lipid deposition through the inflammatory cascade reaction. SOCS1 and SOCS3 are one link in the aforementioned inflammatory network, through which a high-fat diet can affect the progress of disease development; therefore, it is important to clarify the role of SOCS proteins in atherosclerosis. In addition, in non-CHD human populations, we observed that SOCS3 mRNA expression in PBMCs positively correlated with total serum cholesterol levels, which further suggests that a high-fat diet is an important factor in the induction of SOCS3 protein expression. With the increase in cholesterol levels, the expression of SOCS3 also increased. In the circulation, SOCS3 mRNA could only be observed in the PBMCs when the cholesterol reached a certain level $(\sim 100 \mathrm{mg} / \mathrm{dl})$. We can therefore hypothesize that the SOCS3 protein, as a pro-inflammatory cytokine, may mediate inflammatory activation in response to high cholesterol levels; that is, high cholesterol plays a role in pro-inflammation by stimulating SOCS3 expression. This theory has never been mentioned in previous studies. There was no correlation observed between SOCS1, cholesterol and TG in our human study population, which was consistent with our findings in the animal experiments; the effect of a high-fat diet on SOCS1 expression cannot be summarized using a single linear correlation.

When analyzing mouse aortic RNA, we found that the mRNA expression levels of IL-6 but not those of TNF- $\alpha$ significantly increased when the mice were exposed to high cholesterol levels. The expression of IL-6, a pro-inflammatory cytokine thought to be most closely associated with chronic inflammation caused by metabolic abnormalities, was increased continuously with prolonged exposure to high cholesterol. This change in the expression levels of this indicator suggests that as the effect of hypercholesterolemia accumulated, vascular inflammation also increased. Together with our observation of SOCS3, this suggests that the SOCS3 protein in $\mathrm{ApoE}^{-/-}$mice with atherosclerosis may be regulated by the induction of IL-6 in the disease. Li et al (35) found that free cholesterol-loaded mouse peritoneal macrophages are an abundant resource of TNF- $\alpha$ and IL-6. In addition, Frisdal et al (36) showed that lipid loading in human macrophages was accompanied by a strong increase of IL- 6 secretion, followed by the activation of the JAK2/STAT3 signaling pathway by IL- 6 to reduce lipid accumulation. Taking into account our results, as well as those of the above studies, we hypothesized that the high level of cholesterol increased IL-6 expression, subsequently activating JAK2/STAT3. In addition, SOCS3, as a classic negative regulator of JAK2/STAT3, was upregulated with the progression of inflammation aggravated by hyperlipidemia.

By examining the changes in aortic SOCS1 and SOCS3 expression in $\mathrm{ApoE}^{-/-}$mouse models exposed to high cholesterol for different lengths of time, this study observed the differential expression patterns of SOCS1 and SOCS3 in plaque. These results suggest that SOCS1 and SOCS3 play different roles in the development of atherosclerosis in $\mathrm{ApoE}^{-/-}$mice. SOCS3 induced by IL-6 upregulated during hyperlipidemia, may promote the development of atherosclerosis. It is of great importance that we clarify the specific mechanisms of action of SOCS proteins in atherosclerosis.

\section{Acknowledgements}

This study was supported by the Natural Science Foundation of China (30570732 and 30871043 to Z.Y. and 30700320 to Y.L.), the National Science Fund for Distinguished Young Scholars (81025002 to Z.Y.). 


\section{References}

1. Ross R: Atherosclerosis - an inflammatory disease. N Engl J Med 340: 115-126, 1999.

2. Tedgui A and Mallat Z: Cytokines in atherosclerosis: pathogenic and regulatory pathways. Physiol Rev 86: 515-581, 2006.

3. Ortiz-Munoz G, Martin-Ventura JL, Hernandez-Vargas P, et al: Suppressors of cytokine signaling modulate JAK/STAT-mediated cell responses during atherosclerosis. Arterioscler Thromb Vasc Biol 29: 525-531, 2009.

4. Yan C, Cao J, Wu M, et al: Suppressor of cytokine signaling 3 inhibits LPS-induced IL-6 expression in osteoblasts by suppressing CCAAT/enhancer-binding protein \{beta\} activity. J Biol Chem 285: 37227-37239, 2010.

5. Albanesi C, Fairchild HR, Madonna S, et al: IL-4 and IL-13 negatively regulate TNF-alpha- and IFN-gamma-induced betadefensin expression through STAT-6, suppressor of cytokine signaling (SOCS)-1, and SOCS-3. J Immunol 179: 984-992, 2007.

6. Alexander WS and Hilton DJ: The role of suppressors of cytokine signaling (SOCS) proteins in regulation of the immune response. Annu Rev Immunol 22: 503-529, 2004.

7. Yoshimura A, Naka T and Kubo M: SOCS proteins, cytokine signalling and immune regulation. Nat Rev Immunol 7: 454-465, 2007.

8. Davey GM, Heath WR and Starr R: SOCS1: a potent and multifaceted regulator of cytokines and cell-mediated inflammation. Tissue Antigens 67: 1-9, 2006.

9. Egan PJ, Lawlor KE, Alexander WS and Wicks IP: Suppressor of cytokine signaling-1 regulates acute inflammatory arthritis and $\mathrm{T}$ cell activation. J Clin Invest 111: 915-924, 2003.

10. Metcalf D, Di Rago L, Mifsud S, et al: The development of fatal myocarditis and polymyositis in mice heterozygous for IFN-gamma and lacking the SOCS-1 gene. Proc Natl Acad Sci USA 97: 9174-9179, 2000.

11. Ilangumaran S, Ramanathan S and Rottapel R: Regulation of the immune system by SOCS family adaptor proteins. Semin Immunol 16: 351-365, 2004.

12. Jo D, Liu D, Yao S, et al: Intracellular protein therapy with SOCS3 inhibits inflammation and apoptosis. Nat Med 11: 892-898, 2005

13. Li Y, Chu N, Rostami A and Zhang GX: Dendritic cells transduced with SOCS-3 exhibit a tolerogenic/DC2 phenotype that directs type $2 \mathrm{Th}$ cell differentiation in vitro and in vivo. $\mathrm{J}$ Immunol 177: 1679-1688, 2006.

14. Matsumura Y,Kobayashi T, Ichiyama K, et al: Selective expansion of foxp3-positive regulatory $\mathrm{T}$ cells and immunosuppression by suppressors of cytokine signaling 3-deficient dendritic cells. J Immunol 179: 2170-2179, 2007.

15. Liu Y, Stewart KN, Bishop E, et al: Unique expression of suppressor of cytokine signaling 3 is essential for classical macrophage activation in rodents in vitro and in vivo. J Immunol 180 6270-6278, 2008

16. O'Keefe GM, Nguyen VT, Ping Tang LL and Benveniste EN: IFN-gamma regulation of class II transactivator promoter IV in macrophages and microglia: involvement of the suppressors of cytokine signaling-1 protein. J Immunol 166: 2260-2269, 2001.

17. Croker BA, Krebs DL, Zhang JG, et al: SOCS3 negatively regulates IL-6 signaling in vivo. Nat Immunol 4: 540-545, 2003.

18. Yoshimura A, Suzuki M, Sakaguchi R, et al: SOCS, inflammation, and autoimmunity. Front Immunol 3: 20, 2012.

19. Wormald $S$ and Hilton DJ: Inhibitors of cytokine signal transduction. J Biol Chem 279: 821-824, 2004.

20. Brown MS and Goldstein JL: Lipoprotein metabolism in the macrophage: implications for cholesterol deposition in atherosclerosis. Annu Rev Biochem 52: 223-261, 1983.
21. Gower RM, Wu H, Foster GA, et al: CD11c/CD18 expression is upregulated on blood monocytes during hypertriglyceridemia and enhances adhesion to vascular cell adhesion molecule-1. Arterioscler Thromb Vasc Biol 31: 160-166, 2011.

22. Gokalp D, Tuzcu A, Bahceci M, et al: Levels of proinflammatory cytokines and hs-CRP in patients with homozygous familial hypercholesterolaemia. Acta Cardiol 64: 603-609, 2009

23. Sima AV, Stancu CS and Simionescu M: Vascular endothelium in atherosclerosis. Cell Tissue Res 335: 191-203, 2009.

24. Steinberg D: Atherogenesis in perspective: hypercholesterolemia and inflammation as partners in crime. Nat Med 8: 1211-1217, 2002.

25. Tian Y, Yuan Z, Liu Y, et al: Pioglitazone modulates the balance of effector and regulatory $\mathrm{T}$ cells in apolipoprotein $\mathrm{E}$ deficient mice. Nutr Metab Cardiovasc Dis 21: 25-32, 2011.

26. Anderson JL, Adams CD, Antman EM, et al: ACC/AHA 2007 guidelines for the management of patients with unstable angina/ non-ST-Elevation myocardial infarction: a report of the American College of Cardiology/American Heart Association Task Force on Practice Guidelines (Writing Committee to Revise the 2002 Guidelines for the Management of Patients With Unstable Angina/ Non-ST-Elevation Myocardial Infarction) developed in collaboration with the American College of Emergency Physicians, the Society for Cardiovascular Angiography and Interventions, and the Society of Thoracic Surgeons endorsed by the American Association of Cardiovascular and Pulmonary Rehabilitation and the Society for Academic Emergency Medicine. J Am Coll Cardiol 50: el-e157, 2007.

27. Zhao Y, Yuan Z, Liu Y, et al: Activation of cannabinoid CB2 receptor ameliorates atherosclerosis associated with suppression of adhesion molecules. J Cardiovasc Pharmacol 55: 292-298, 2010.

28. Xue JH, Yuan Z, Wu Y, et al: High glucose promotes intracellular lipid accumulation in vascular smooth muscle cells by impairing cholesterol influx and efflux balance. Cardiovasc Res 86: $141-150,2010$.

29. He L, He M, Lv X, et al: NF-kappaB binding activity and proinflammatory cytokines expression correlate with body mass index but not glycosylated hemoglobin in Chinese population. Diabetes Res Clin Pract 90: 73-80, 2010.

30. Tang J, Kozaki K, Farr AG, et al: The absence of platelet-derived growth factor-B in circulating cells promotes immune and inflammatory responses in atherosclerosis-prone $\mathrm{ApoE}^{-/-}$mice. Am J Pathol 167: 901-912, 2005.

31. Endo TA, Masuhara M, Yokouchi M, et al: A new protein containing an SH2 domain that inhibits JAK kinases. Nature 387: 921-924, 1997.

32. Park SJ, Oh EJ, Yoo MA and Lee SH: Involvement of DNA-dependent protein kinase in regulation of stress-induced JNK activation. DNA Cell Biol 20: 637-645, 2001

33. Kinjyo I, Hanada T, Inagaki-Ohara $\mathrm{K}$, et al: SOCS1/JAB is a negative regulator of LPS-induced macrophage activation. Immunity 17: 583-591, 2002.

34. Libby P, Ridker PM and Hansson GK: Inflammation in atherosclerosis: from pathophysiology to practice. J Am Coll Cardiol 54: 2129-2138, 2009.

35. Li Y, Schwabe RF, DeVries-Seimon T, et al: Free cholesterolloaded macrophages are an abundant source of tumor necrosis factor-alpha and interleukin-6: model of NF-kappaB- and map kinase-dependent inflammation in advanced atherosclerosis. J Biol Chem 280: 21763-21772, 2005.

36. Frisdal E, Lesnik P, Olivier M, et al: Interleukin-6 protects human macrophages from cellular cholesterol accumulation and attenuates the proinflammatory response. J Biol Chem 286 : 30926-30936, 2011. 